Y. GOTOH

KODAI MATH. J.

16 (1993), 79-89

\title{
REMARKS ON MULTIPLIERS FOR BMO ON GENERAL DOMAINS
}

Dedicated to Profssor Nobuyuki Suita on his sixtieth birthday

BY YASUHIRO GOTOH

\section{Introduction.}

A measurable function $\varphi$ is called a (pointwise) $B M O$ multiplier if $\phi f \in B M O$ for every $f \in B M O$. The characterizations of multipliers for $B M O$ spaces on $n$ dimensional torus $\boldsymbol{T}^{n}$ and on $n$ dimensional Euclidean space $\boldsymbol{R}^{n}$ are well known (Stegenga [9], Janson [4], Nakai-Yabuta [6]).

Although Nakai-Yabuta's characterization of $B M O\left(\boldsymbol{R}^{n}\right)$ multiplier is more complicate than that of $B M O\left(\boldsymbol{T}^{n}\right)$, these characterizations are essentially the same. Indeed we shall give a geometrically simple characterization of $B M O(D)$ multiplier for general domain $D$ in $\boldsymbol{R}^{n}$ by using a metric on a space of some family of cubes in $D$, which is also valid for $B M O\left(\boldsymbol{T}^{n}\right)$.

\section{§ 1. Preliminary and main result.}

Throughout this paper we treat only 2 dimensional case for the simplicity, since the same argument holds in the case of general dimension. Let $D$ be a domain lying in $\boldsymbol{R}^{2}$ and $f \in L_{l o c}^{1}(D)$. We say $f \in B M O(D)$ if

$$
\|f\|_{*}=\|f\|_{*, D}=\sup _{Q} \frac{1}{m(Q)} \int_{Q}\left|f-f_{Q}\right| d m<\infty,
$$

where $d m$ is the two dimensional Lebesgue measure, $f_{Q}=\left.m(Q)^{-1}\right|_{J_{Q}} f d m$ and the supremum is taken for all closed squares $Q$ in $D$ whose sides are paralleel to the coordinate axes.

We recall some notations and results in our former paper [3]. From now on 'square' means a closed square whose sides parallel to the coordinate axes, 'dyadic square' means a square $\left[k 2^{n},(k+1) 2^{n}\right] \times\left[l 2^{n},(l+1) 2^{n}\right], k, l, n \in \boldsymbol{Z}, l(Q)$ denotes the side length of a square $Q, t Q, t>0$, denotes the square having the same center as $Q$ and $t l(Q)$ as its side length, $d(, \cdot)$ denotes the Euclidean distance, $A>0$ denotes a universal constant which may vary from place to place. We say that a square $Q$ lying in $D$ is admissible if it satisfies $d(Q, \partial D) \geqq 32 l(Q)$ and $\mathcal{A}(D)$ denotes the set of all admissible squares in $D$. A sequence of admissible square $Q_{0}, Q_{1}, \cdots, Q_{n}$ in $D$ satisfying the condition

Received May 14, 1992. 


$$
\begin{array}{ll}
Q_{i} \cap Q_{i+1} \neq \emptyset, & 0 \leqq i \leqq n-1, \\
\frac{1}{2} \leqq \frac{l\left(Q_{i}\right)}{l\left(Q_{\imath+1}\right)} \leqq 2, & 0 \leqq i \leqq n-1,
\end{array}
$$

is called an admissible chain. Let $Q, Q^{\prime}$ be two admissible squares in $D$. We define

$$
\delta_{D}\left(Q, Q^{\prime}\right)=\min \left\{n \geqq 1 \mid Q=Q_{0}, Q_{1}, \cdots, Q_{n}=Q^{\prime} \text { is an admessible chain }\right\}
$$

and the admissible chain which attains above minimum is called geodesic admissible chain joining $Q$ and $Q^{\prime}$. Since we define $\delta_{D}$ so that $\delta_{D} \geqq 1$ by technical reason, $\delta_{D}$ is not a distance function, but the triangle inequality holds. In [3] we have used $\delta_{D}$ to characterize the domain with 'relative' $B M O$ extension property. Let $Q, Q^{\prime} \in \sum \mathcal{A}\left(\boldsymbol{R}^{2}\right)$, that is, $Q, Q^{\prime}$ be arbitrary squares lying in $R^{2}$. We define

$$
\psi\left(Q, Q^{\prime}\right)=\log \left(1+\frac{l(Q)+l\left(Q^{\prime}\right)+d\left(Q, Q^{\prime}\right)}{l(Q)}\right)\left(1+\frac{l(Q)+l\left(Q^{\prime}\right)+d\left(Q, Q^{\prime}\right)}{\mathrm{WO}}\right),
$$

then

PROPOSITION 1. ([3]) Let $Q, Q^{\prime} \in \mathcal{A}(D)$ then

$$
\phi\left(Q, Q^{\prime}\right) \leqq A \delta_{D}\left(Q, Q^{\prime}\right)
$$

Conversely if there exists a square $\tilde{Q}$ such that $Q \cup Q^{\prime} \subset \tilde{Q} \subset \tilde{Q} \subset \subset D$ then

$$
\delta_{D}\left(Q, Q^{\prime}\right) \leqq A \psi\left(Q, Q^{\prime}\right) .
$$

Especially, for all squares $Q, Q^{\prime} \in \mathcal{A}\left(\boldsymbol{R}^{2}\right)$, we have

$$
A^{-1} \phi\left(Q, Q^{\prime}\right) \leqq \delta_{R^{2}}\left(Q, Q^{\prime}\right) \leqq A \psi\left(\measuredangle 1, Q^{\prime}\right)
$$

Let $D$ be a proper subdomain of $\boldsymbol{R}^{2}$. There exists a decomposition of $D$ into a countable family of dyadic squares $\mathscr{D}(D)=\left\{Q_{\lambda}\right\}, Q_{\lambda}{ }^{\circ} \cap Q_{\mu}{ }^{\circ}=\emptyset,(\lambda \neq \mu)$, $\cup_{\lambda} Q_{\lambda}=D$ such that

$$
32 \leqq \begin{aligned}
& d\left(Q_{\lambda}, \partial D\right) \\
& l\left(Q_{\lambda}\right)
\end{aligned} \leqq 66
$$

which we call Whitney decomposition of $D$. We say that a sequence $Q_{0}, Q \downarrow$, $\cdots, Q_{n} \in \mathscr{D}(D)$ is a Whitney chain if $Q_{i} \cap Q_{\imath+1} \neq \emptyset$. Since $\mathscr{D}(D) \subset \mathcal{A}(D)$, every Whitney chain is admissible. Let $Q, Q^{\prime} \in \mathscr{D}(D)$. We set

$$
W_{D}\left(Q, Q^{\prime}\right)=\min \left\{n \geqq 1 \quad Q=Q_{0}, Q_{1}, \cdots, Q_{n}=Q^{\prime} \text { is a Whitney chain }\right\}
$$

and the Whitney chain which attains above minimum is called geodesic Whitney chain joining $Q$ and $Q^{\prime}$. It holds that $\delta_{D}\left(Q, Q^{\prime}\right) \leqq W_{D}\left(Q, Q^{\prime}\right), Q, Q^{\prime} \in \mathscr{D}(D)$ by definition. Conversely

PROPOSITION 2. ([3]) $W_{D}\left(Q, Q^{\prime}\right) \leqq A \delta_{D}\left(Q, Q^{\prime}\right), Q, Q^{\prime} \in \mathscr{D}(D)$. 
Let $Q_{0} \in \mathscr{D}(D)$ and set

$$
f(z)=W_{D}\left(Q, Q_{0}\right), \quad z \in Q \in \mathscr{D}(D) .
$$

Then $f \in B M O(D)$ and $\|f\|_{*} \leqq A$, which is a consequence of the following localization theorem.

Proposition 3. (cf. Reimann-Rychener [7], Jones [5]) Let $\lambda \geqq 1$. Let $f$ be a function in $L_{\text {loc }}^{1}(D)$ satisfying the condition

$$
\frac{1}{m(Q)} \int_{Q}\left|f-f_{Q}\right| d m \leqq K
$$

for every square $Q$ in $D$ such that $d(Q, \partial D) \geqq \lambda l(Q)$ then $f \in B M O(D)$ and $\|f\|_{*, D}$ $\leqq A K \lambda$.

$B M O(D)$ functions induce Lipschitz continuous functioins on $\mathcal{A}(D)$ as follows ;

PROPOSITION 4. ([3]) Let $D$ be arbitrary domain and $f \in B M O(D)$. Then

$$
\left|f_{Q}-f_{Q^{\prime}}\right| \leqq A\|f\|_{*, D} \delta_{D}\left(G_{Q}\right), \quad Q, Q^{\prime} \in \mathcal{A}(D) .
$$

Let $D$ be arbitrary domain, $Q_{0} \in \mathcal{A}(D)$ and $f \in B M O(D)$. We say $/ \epsilon$ $V M O(D)$ if

$$
\frac{1}{m(Q)} \int_{Q}\left|f-f_{Q}\right| d m \longrightarrow 0
$$

as $Q \in \mathcal{A}(D), \delta_{D}\left(Q, Q_{0}\right)-+\infty$. Every continuous function on $D$ with compact support belongs to $V M O(D)$. $V M O(D)$ is a closed subspace of $B M O(D)$ and its definition is independent of the choice of $Q_{0} \in \mathcal{A}(D)$. When $D$ is bounded, $f \in$ $V M O(D)$ if and only if $f \in B M O(D)$ and $m(Q)^{-1}|| f-f_{Q} \mid d m \rightarrow$ as $Q \in \mathcal{A}(D)$, $l(Q) \rightarrow 0 . \quad B M O(D)$ and $V M O(D)$ are invariant under quasi-conformal mappings. Remark that in case of $D=\boldsymbol{R}^{2}$, our $V \cdot M O\left(\boldsymbol{R}^{2}\right)$ space does not coinside with the usual $V M O$ space which consists of function $f \in B M O\left(\boldsymbol{R}^{2}\right)$ such that $m(Q)^{-1} \int_{J_{Q}}\left|f-f_{Q}\right| d m \rightarrow 0$ as $Q \in \mathcal{A}(D), l(Q) \rightarrow 0$. It is easy to show that our VMO space on $\boldsymbol{R}^{2}$ is contained in the usual $V M O$ space on $\boldsymbol{R}^{2}$, but the converse is not true. For example, $\log ^{+}|z|$ belongs to the usual $V M O$ space on $R^{2}$ but it does not belong to our $V M O$ space on $\boldsymbol{R}^{2}$.

By using the same method as the proof of Proposition 4, we have

PROPOSITION 4'. Let $D$ be arbitrary domain and $f \in V M O(D)$. Then

$$
\left|f_{Q}-f_{Q_{0}}\right|=o\left(\delta_{D}\left(Q, Q_{0}\right)\right)
$$

as $Q \in \mathcal{A}(D), \delta_{D}\left(Q, Q_{0}\right) \rightarrow \infty$.

We say a measurable function $\phi$ is a $B M O(D)$ (resp. $V M O(D))$ multiplier if $\phi f \in B M O(D)(V M O(D))$ for every $f \in B M O(D)(V M O(D))$. To consider $B M O$ or 
$V M O$ multiplier it is convenient to introduce the norm

$$
\|f\|_{* *}=\|f\|_{* *, D}=\|f\|_{*, D}+|f|_{Q 0}, \quad f \in B M O(D)(V M O(D))
$$

where $Q_{0}$ is a fixed square in $\mathcal{A}(D)$ and $|f|_{Q_{0}}=m\left(Q_{0}\right)^{-S_{Q_{0}}}|f| d m$. Then closed graph theorem shows that the operator $T_{\phi}: f \mapsto \phi f$ on $B M O(D)\left(T_{\phi}^{\prime}: f \mapsto \phi f\right.$ on $V M O(D))$ is bounded. Let $\left\|T_{\phi}\right\|\left(\left\|T_{\phi}^{\prime}\right\|\right)$ denotes its operator norm. Our main result is

THEOREM 1. Let $D$ be arbitrary domain. For a measurable function $\phi$ on $D$, the following three conditions are equivalent to each other

(1) $\varphi$ is a $B M O(D)$ multiplier.

(2) $\varphi$ is a $V M O(D)$ multiplier.

(3) There exists a constant $M \geqq 0$ such that

$$
\begin{gathered}
\|\phi\|_{\infty} \leqq M, \\
\frac{1}{m(Q)} \int_{Q}\left|\phi-\phi_{Q}\right| d m \leqq \frac{M}{\delta_{D}\left(Q, Q_{0}\right)}, \quad Q \in \mathcal{A}(D) .
\end{gathered}
$$

In this case $\left\|T_{\phi}\right\| \leqq A M,\left\|T_{\phi}^{\prime}\right\| \leqq A M$ holds. Conversely if $\varphi$ is a $B M O(D)$ (resp. $V M O(D))$ multiplier then we can choose the constant $M$ so that $M \leqq A\left\|T_{\phi}\right\|(M \leqq$ $\left.A\left\|T_{\phi}^{\prime}\right\|\right)$.

COROLLARY 1. (cf. Nakai-Yabuta [6]) For a measurable functions $\varphi$ on $\boldsymbol{R}^{2}$ the following conditions are equivalent to each other

(1) $\varphi$ is a $B M O\left(\boldsymbol{R}^{2}\right)$ multiplier.

(2) $\varphi$ is a $V M O\left(\boldsymbol{R}^{2}\right)$ multiplier.

(3) $\phi \in L^{\infty}\left(\boldsymbol{R}^{2}\right)$ and there exists a constant $M \geqq 0$ such that

$$
\frac{1}{m(Q)} \int_{Q}\left|\phi-\phi_{Q}\right| d m \leqq \frac{M}{\phi\left(Q, Q_{0}\right)}
$$

for every square $Q$ in $\boldsymbol{R}^{2}$.

Moreover we can replace $\boldsymbol{R}^{2}$ with arbitrary inner NTA domain, especially arbitrary uniform domain (see $\$ 3$ ), in this corollary.

Let $\Delta=\{|z|<1\}$. Since $\delta\left(Q, Q_{0}\right), Q \in \mathcal{A}(\Delta)$ is comparable with $\log (2+(1 / l(Q))$, we have the following by Theorem 1 and Proposition 3 (of disk version). Its correspondence for holomorphic $B M O$ function, which is usually called Bloch function, is well known (Brown-Shields [1]).

COROLLARY 2. For a measurable functions $\varphi$ on $A$ the following conditions are equivalent to each other

(1) $\varphi$ is a $B M O(\Delta)$ multiplier.

(2) $\varphi$ is a $V M C(\Delta)$ multiplier.

(3) $\phi \in L^{\infty}(\Delta)$ and there exists a constant $M \geqq 0$ such that 


$$
\frac{1}{m(B)} \int_{B}\left|\phi-\phi_{B}\right| d m \leqq M\left(\log \left(2+\frac{1}{\operatorname{rad}(B)}\right)\right)^{-1}
$$

for every disk $B$ in $\Delta$, where $\operatorname{rad}(B)$ denotes the radius of $B$.

Moreover we can also replace $\Delta$ with arbitrary Holder domain, especially arbitrary bounded uniform domain (see $\$ 3$ ), in this corollary.

Let $S$ be the unit sphere in $R^{3}, \sigma$ the normalized surface measure on $S$ and $B M O_{\sigma}(\boldsymbol{S})$ the $B M O$ space on $S$ with respect to $\sigma$. We fix a disk $B_{0}$ on $S$. Then the distance between $B_{0}$ and arbitrary ball $B$ on $S$, which corresponds to $\delta_{D}$, is comparable with $\log (2+(1 / \operatorname{rad}(B))$. Hence we have the following result, its one dimensional version for the $B M O$ space on the unit circle is well known (Stegenga [9], Janson [4]), as the same way.

COROLLARY 3. For a measurable functions $\phi$ on $S$ the following conditions are equivalent to each other

(1) $\varphi$ is a $B M O_{\sigma}(\boldsymbol{S})$ multiplier.

(2) $\varphi$ is a $V M O_{\sigma}(\boldsymbol{S})$ multiplier.

(3) $\phi \in L^{\infty}(\boldsymbol{S})$ and there exists a constant $M \geqq 0$ such that

$$
\frac{1}{\sigma(B)} \int_{B}\left|\phi-\phi_{B, \sigma}\right| d \sigma \leqq M\left(\log \left(2+\frac{1}{\operatorname{rad}(B)}\right)\right)^{-1}
$$

for every disk $B$ in $\boldsymbol{S}$.

Since we can identify $B M O\left(\boldsymbol{R}^{2}\right)$ and $V M O\left(\boldsymbol{R}^{2}\right)$ with $B M O_{\sigma}(\boldsymbol{S})$ and $V M O_{\sigma}(\boldsymbol{S})$ respectively throughout the stereographic projection (See Reimann-Rychener [7] for $B M O$, and the similar method proves this for $V M O$.), Corollary 3 gives another characterization of $B M O\left(\boldsymbol{R}^{2}\right)$ multiplers.

\section{$\S 2$. Proof of Theorem 1 .}

LEMMA 1. (cf. Stegenga [9], Nakai-Yabuta [6]) // $\varphi$ is a $B M O(D)$ (resp. $V M O(D))$ multiplier then $\phi \in L^{\infty}(D)$ and $\|\phi\|_{\infty} \leqq 3\left\|T_{\phi}\right\|\left(\|\phi\|_{\infty} \leqq 3\left\|T_{\phi}^{\prime}\right\|\right)$.

Proof. Let $\varphi$ be a $V M O(D)$ multiplier. We fix a point $z \in D$. Let $Q$ be the square having $z$ as its center and $l(Q)=t$. Let $h$ be a function on $D$ such that

$$
|h|={\underset{m(\psi)}{1}}_{x_{Q},} \chi_{J \nu} h d m=0
$$

and set $k=\overline{\operatorname{sgn}}(\phi h)$. Let $k_{n}$ be a sequence of continuous function with compact support which converges to $k$ a.e. and $\left\|k_{n}\right\|_{\infty} \leqq\|k\|_{\infty}$. Then $k_{n} \in V M O(D)$ and since $\left\|k_{n}\right\|_{\infty} \leqq 1$ we have $\left\|k_{n}\right\|_{* *} \leqq 3$. Hence

$$
\int_{D} k_{n} \phi h d m=\int_{D}\left\{\phi k_{n}-\left(\phi k_{n}\right)_{Q}\right\} h d m
$$




$$
\leqq \frac{1}{m(Q)} \int_{Q}\left|\phi k_{n}-\left(\phi k_{n}\right)_{Q}\right| d m \leqq\left\|k_{n} \phi\right\|_{*} \leqq 3\left\|T_{\phi}^{\prime}\right\|
$$

And so by $n \rightarrow \infty$,

$$
\frac{1}{m(Q)} \int_{Q}|\phi| d m=\int_{D} k \phi h d m \leqq 3\left\|T_{\phi}^{\prime}\right\|
$$

Letting $t \rightarrow 0$ we have $\|\phi\|_{\infty} \leqq 3\left\|T_{\phi}^{\prime}\right\|$ by Lebesgue's theorem. This proves the assertion since above proof is valid for $B M O$.

Q. E. D.

LEMMA 2. (cf. Stegenga [9], Nakai-Yabuta [6]) Let $f \in L_{l o c}^{1}(D)$ and $\phi \in$ $L^{\infty}(D)$ then

$$
|| f_{Q}\left|\frac{1}{m(Q)} \int_{Q}\right| \phi-\phi_{Q}\left|d m-\frac{1}{m(Q)} \int_{Q}\right| \phi f-(\phi f)_{Q}|d m| \leqq 2\|\phi\|_{\infty} \frac{1}{m(Q)} \int_{Q}\left|f-f_{Q}\right| d m .
$$

holds for every square $Q$ lying in $D$.

Proof.

$$
\begin{aligned}
& || f_{Q}\left|\frac{1}{m(Q)} \int_{Q}\right| \phi-\phi_{Q}\left|d m-\frac{1}{m(Q)} \int_{Q}\right| f \phi-(f \phi)_{Q}|d m| \\
& \quad \leqq \frac{1}{m(Q)} \int_{Q}\left(\left|\left(f-f_{Q}\right) \phi\right|+\left|f_{Q} \phi_{Q}-(f \phi)_{Q}\right|\right) d m \\
& \quad \leqq\|\phi\|_{\infty} \frac{1}{m(Q)} \int_{Q}\left|f-f_{Q}\right| d m+\left|\frac{1}{m(Q)} \int_{Q}\left(f-f_{Q}\right) \phi d m\right| \\
& \quad \leqq 2\|\phi\|_{\infty} \frac{1}{m(Q)} \int_{Q}\left|f-f_{Q}\right| d m .
\end{aligned}
$$

The following lemma shows that the estimation of Proposition 4 is best possible.

LEMMA 3. Let $D$ be arbitrary domain and $Q_{0}, Q_{1} \in \mathcal{A}(D)$. Then there exists a function $f \in V M O(D)$ such that

$$
\delta_{D}\left(Q_{0}, Q_{1}\right) \leqq A\left|f_{Q_{1}}\right|+A, \quad\|f\|_{*, D} \leqq A, \quad|f|_{Q_{0}} \leqq A .
$$

Proof. First, assume there exists a square $\tilde{Q}$ in $D$ such that $Q_{0} \cup Q_{1} \subset \tilde{Q} \subset$ $2 \tilde{Q} \subset D$. Let $z_{\imath}, i=0,1$ be the center of $Q_{\imath}$. In this case the first inequality reduces to $\phi_{D}\left(Q_{0}, Q_{1}\right) \leqq A \mid f_{Q_{1}}+A$ by Proposition 1 , and so the function

$$
f(z)=\min \left\{\log ^{+}\left(\frac{l\left(Q_{0}\right)+l\left(Q_{1}\right)+d\left(Q_{0}, Q_{1}\right)}{\left|z-z_{1}\right|}\right), \log \left(\frac{l\left(Q_{0}\right)+l\left(Q_{1}\right)+d\left(Q_{0}, Q_{1}\right)}{l\left(Q_{1}\right)}\right)\right\}
$$

if $l\left(Q_{1}\right)<l\left(Q_{0}\right)$ and

$$
f(z)=\min \left\{\log +\frac{\left|z-z_{0}\right|}{l\left(Q_{0}\right)}, \log \left(\frac{l\left(Q_{0}\right)+l\left(Q_{1}\right)+d\left(Q_{0}, Q_{1}\right)}{l\left(Q_{0}\right)}\right)\right\}
$$


if $l\left(Q_{1}\right) \geqq l\left(Q_{0}\right)$, satisfies the required condition since $\log |z| \in B M O\left(\boldsymbol{R}^{2}\right)$.

Next assume there exists no such square $\tilde{Q}$. In this case $D \neq \boldsymbol{R}^{2}$. Let $Q_{\imath}^{\prime}$, $i=0,1$ be a square in $\mathscr{D}(D)$ such that $Q_{i} \cap Q_{i}^{\prime} \neq \emptyset$ and $z_{\imath}$ the center of $Q_{\imath}$. Then

$$
\begin{aligned}
\delta_{D}\left(Q_{0}, Q_{1}\right) & \leqq \delta_{D}\left(Q_{0}, Q_{0}^{\prime}\right)+\delta_{D}\left(Q_{0}^{\prime}, Q_{1}^{\prime}\right)+\delta_{D}\left(Q_{1}^{\prime}, Q_{1}\right) \\
& \leqq A \log +\frac{l\left(Q_{0}^{\prime}\right)}{l\left(Q_{0}\right)}+A W_{D}\left(Q_{0}^{\prime}, Q_{1}^{\prime}\right)+A \log ^{+} \frac{l\left(Q_{1}^{\prime}\right)}{l\left(Q_{1}\right)}+A
\end{aligned}
$$

Let

$$
\begin{array}{ll}
f_{1}(z)=\min \left\{\log ^{+} \frac{\left|z-z_{0}\right|}{l\left(Q_{0}\right)}, \log ^{+} \frac{l\left(Q_{0}^{\prime}\right)}{l\left(Q_{0}\right)}\right\}, & z \in D, \\
f_{2}^{\prime}(z)=m m\left\{W_{D}\left(Q_{0}^{\prime}, Q\right), W_{D}\left(Q_{0}^{\prime}, Q_{1}^{\prime}\right)\right\}, & z \in Q \in \mathcal{D}(D), \\
f_{3}(z)=\min \left\{\log ^{+} \frac{l\left(Q_{1}^{\prime}\right)}{\left|z-z_{0}\right|}, \log ^{+} \frac{l\left(Q_{1}^{\prime}\right)}{l\left(Q_{1}\right)}\right\}, \quad z \in D,
\end{array}
$$

We slightly modify $f_{2}^{\prime}$ into a continuous functions $f_{2}$ (or we may define $f_{2}$ by

$$
f_{2}(z)=\min \left\{k_{D}\left(z_{0}, z\right), k_{D}\left(z_{0}, z_{1}\right)\right\}
$$

where $z_{\imath}$ be the center of $Q_{\imath}$ and $k_{D}$ is the distance function obtained by the quasi-hyperbolic metric $|d z| / d(z, \partial D))$ and set $f=f_{1}+f_{2}+f_{3}$ Then $f \in V M O(D)$ and by the remark below Proposition 2, we have $\|f\|_{*, D} \leqq \sum_{i=1}^{3}\left\|f_{i}\right\|_{*, D} \leqq A, \quad|f|_{Q_{0}}$ $\leqq \sum_{i=1}^{3}\left|f_{\imath}\right|_{Q_{0}} \leqq A$ and

$$
\log \frac{l\left(Q_{0}^{\prime}\right)}{l\left(Q_{0}\right)} \leqq A\left(f_{1}\right)_{Q_{1}}+A, \quad W_{D}\left(Q_{0}^{\prime}, Q_{1}^{\prime}\right) \leqq A\left(f_{2}\right)_{Q_{1}}+A, \quad \log \frac{l\left(Q_{1}^{\prime}\right)}{l\left(Q_{1}\right)} A\left(f_{3}\right)_{Q_{1}}+A .
$$

Summerizing above inequalities we have $\delta_{D}\left(Q_{0}, Q_{1}\right) \leqq A\left|f_{Q_{1}}\right|+A$. Q. E. D.

Proof of Theorem 1. We will prove only $(1) \leftrightarrow(3)$ since we can show (2) $\leftrightarrow(3)$ similarly by appealing to Proposition $4^{\prime}$ instead of Proposition 4. Let $\varphi$ satisfy the condition (3). Let $f \in B M O(D)$ and $Q \in \mathcal{A}(D)$. Proposition 4 shows that

Hence by Lemma 2,

$$
\begin{aligned}
\left|f_{Q}\right| \frac{1}{m(Q)} \int_{Q}\left|\phi-\phi_{Q}\right| d m & \leqq\left\{\left|f_{Q_{0}}\right|+A\|f\|_{*, D} \delta_{D}\left(Q, Q_{0}\right)\right\} \frac{M}{\delta_{D}\left(Q, Q_{0}\right)} \\
& \leqq A M\left\{|f|_{Q_{0}}+\|f\|_{*, D}\right\} \leqq A M\|f\|_{* *, D} .
\end{aligned}
$$

$$
\frac{1}{m(Q)} \int_{Q}\left|\phi f-(\phi f)_{Q}\right| d m \leqq A M\|f\|_{* *, D}+2\|\phi\|_{\infty}\|f\|_{*} \leqq A M\|f\|_{* *, D} .
$$

Applying localization theorem we have $\|\phi f\|_{*, D} \leqq A M\|f\|_{* *, D}$. Since $|\phi f|_{Q_{0}} \leqq$ $\|\phi\|_{\infty}|f|_{Q_{0}} \leqq M\|f\|_{* *, D}$ it follows that $\|\phi f\|_{* *, D} \leqq A M\|f\|_{* *, D}$.

Conversely let $\varphi$ be a $B M O(D)$ multiplier. Let $Q_{1} \in \mathcal{A}(D), f$ the function satisfying the condition of Lemma 3. Then Lemmas 1 and 2 show that 


$$
\begin{gathered}
\delta_{D}\left(Q_{1}, Q_{0}\right) \frac{1}{m} \frac{1}{\left(Q_{1}\right)} \int_{Q_{1}}\left|\phi-\phi_{Q_{1}}\right| d m \leqq\left(A\left|f_{Q_{1}}\right|+A\right) \frac{1}{m\left(Q_{1}\right)} \int_{Q_{1}}\left|\phi-\phi_{Q_{1}}\right| d m \\
\leqq A\left(\left\|T_{\phi} f\right\|_{*, D}+2\|\phi\|_{\infty}\|f\|_{*}\right)+A\left\|T_{\phi} 1\right\|_{*, D} \leqq A\left\|T_{\phi}\right\|
\end{gathered}
$$

which implies the assertion.

Q. E. D.

\section{$\S 3$. Some consequences.}

Let $D \neq \boldsymbol{R}^{2}$. We say a function $F$ on $\mathscr{D}(D)$ is admissible if it satisfies $F \geqq M^{-1}$ and

$$
M^{-1} \leqq \frac{F(Q)}{F\left(Q^{\prime}\right)} \leqq M, \quad Q \cap Q^{\prime} \neq \emptyset, \quad Q, Q^{\prime} \in \mathscr{D}(D)
$$

for some constant $M>0$. Let $\mathrm{F}$ be an admissible function on $\mathscr{D}(D)$. We set

$$
\hat{F}(Q)=F(\tilde{Q})+\log \left(2+\frac{l(\tilde{Q})}{l(Q)}\right), \quad Q \in \mathcal{A}(D)
$$

where $\tilde{Q}$ is one of the square in $\mathscr{D}(D)$ such that $\tilde{Q} \cap Q \neq \emptyset$. We fix a square $Q_{0} \in \mathcal{A}(D)$.

THEOREM 2. Let $D \neq \boldsymbol{R}^{2}$. The following conditions are equivalent for an admissible function $F$ on $\mathscr{D}(D)$

(1) There exists a constant $M>0$ such that

$$
\delta_{D}\left(Q, Q_{0}\right) \leqq M \hat{F}(Q), \quad Q \in \mathcal{A}(D) .
$$

(2) Let $\phi$ be an $L^{\infty}(D)$ function on $D$ satisfyingthe condition

$$
\frac{1}{m(Q)} \int_{Q}\left|\phi-\phi_{Q}\right| d m \leqq \frac{M}{\hat{F}(Q)}, \quad Q \in \mathcal{A}(D)
$$

for some constant $M \geqq 0$, then $\varphi$ is a $B M O(D)$ multiplier.

(3) Let $\varphi$ be an $L^{\infty}(D)$ function on $D$ satisfying the same condition as (2) then $\varphi$ is a $V M O(D)$ multiplier.

Proof. (2) $\leftrightarrow(3)$ and $(1) \rightarrow(2)$ are the consequence of Theorem 1. Now will prove $(2) \rightarrow(1)$. We can assume $Q_{0} \in \mathscr{D}(D)$ from the beginning since the condition (1) is independent of the choice of $Q_{0} \in \mathcal{A}(D)$. Then $\delta_{D}\left(Q, Q_{0}\right), Q \in \mathcal{A}(D)$ is comparable with $W_{D}\left(\tilde{Q}, Q_{0}\right)+\log (2+(l(\tilde{Q}) / l(Q))$ where $\tilde{Q}$ is one of the squares in $\mathscr{D}(D)$ such that $Q \cap \tilde{Q} \neq \emptyset$. Let $h$ be a fixed non-zero $C^{\infty}$ function supported on the square of side length 1 and center the origin such that $\int_{j} h d m=0$. Let $Q \in$ $\mathscr{D}(D)$ and $z_{0}$ its center. We set a function $\varphi$ on $D$ by

$$
\phi(z)=\frac{1}{F(Q)} h\left(\frac{z-z_{0}}{l(Q)}\right) \quad z \in Q \in \mathscr{D}(D) .
$$


$\phi$ is a bounded $C^{\infty}(D)$ function on $D$ and it holds that

$$
|\nabla \phi(z)| \leqq \frac{A}{F(Q) l(Q)}, \quad z \in Q \in \mathscr{D}(D) .
$$

Let $Q \in \mathcal{A}(D)$. Let $\tilde{Q}$ one of the square in $\mathscr{D}(D)$ such that $Q \cap \tilde{Q} \neq \emptyset$ and $z_{0}$ its center then by above estimate we have

$$
\begin{aligned}
\frac{1}{m(Q)} \int_{Q}\left|\phi-\phi_{Q}\right| d m & \leqq \frac{2}{m(Q)} \int_{Q}\left|\phi-\phi\left(z_{0}\right)\right| d m \\
& \leqq \frac{2}{m(Q)} \int_{Q} \frac{A}{F(\tilde{Q}) l(\tilde{Q})} l(Q) d m \leqq \frac{A}{F(\tilde{Q})} \frac{l(Q)}{l(\tilde{Q})} \leqq \frac{A}{\hat{F}(Q)}
\end{aligned}
$$

Hence $\phi$ is a $B M O$ multiplier by the assumption. Further let $Q \in \mathscr{D}(D)$ then

$$
\frac{1}{m(Q)} \int_{Q}\left|\phi-\phi_{Q}\right| d m=\frac{1}{m(Q)} \int_{Q}|\phi| d m \geqq \frac{A}{F(Q)}
$$

and so theorem 1 implies the assertion.

Q. E. D.

Let $Q_{0} \in \mathcal{A}(D)$. We say a domain D is an inner NTA domain if there exists a constant $M>0$ such that

$$
\delta_{D}\left(Q, Q_{0}\right) \leqq M \psi\left(Q, Q_{0}\right), \quad Q \in \mathcal{A}(D) .
$$

This deefinition is somewhat different from the original one (cf. Shimomura [8]). $\boldsymbol{R}^{2}$ is inner NTA by Proposition 1. More generally every uniform domain is inner NTA (cf. Gehring [2]). We say also a domain $D$ is a Holder domain (cf. Shimomura [8]) if there exists a constant $M>0$ such that

$$
\delta_{D}\left(Q, Q_{0}\right) \leqq M \log \left(2+\frac{1}{l(Q)}\right), \quad Q \in \mathcal{A}(D) .
$$

These definitions are independent of the choice of $Q_{0} \in \mathcal{A}(D)$. Remark that the inverse inequality holds for every domain in either case. In case of $D \neq \boldsymbol{R}^{2}$, $D$ is inner NTA if and only if there exists a square $Q_{0} \in \mathscr{D}(D)$ and a constant $M>0$ such that

$$
W_{D}\left(Q, Q_{0}\right) \leqq M \psi\left(Q, Q_{0}\right), \quad Q \in \mathscr{D}(D)
$$

and $D$ is Holder if and only if there exists a square $Q_{0} \in \mathscr{D}(D)$ and a constant $M>0$ such that

$$
W_{D}\left(Q, Q_{0}\right) \leqq M \log \left(2+\frac{1}{l(Q)}\right) \quad Q \in \mathscr{D}(D) .
$$

There is a simple relation between inner NTA domains and Holder domains.

LEMMA 4. A domain $D$ is a Holder domain if and only if it is a bounded inner NTA domain. 
Proof. It suffices to show that Holder domains are bounded. Let $D$ be a Holder domain. Since $l(Q) \rightarrow 0$ as $Q \in \mathcal{A}(D), Q \rightarrow \infty, D \neq \boldsymbol{R}^{2}$ and we can assume that $Q_{0}$ is the biggest square in $\mathscr{D}(D)$ and $l\left(Q_{0}\right)=1$ Let $Q \in \mathscr{D}(D)$ and set $l(Q)$ $=2^{-N}$. Let $Q_{0}, Q_{1}, \cdot \cdot, Q_{n}=Q$ be a geodesic Whitney chain and set $n_{0}=0, n_{k}=$ $\max \left\{n \mid l\left(Q_{n}\right)=2^{-k}\right\}, 1 \leqq k \leqq N$. Let $z_{k}$ be the center of $Q_{n_{k}}$ and set $d_{k}=\left|z_{k}-z_{k-1}\right|$. Since $n_{k}-n_{k-1} \geqq A 2^{k} d_{k}$ we have

$$
\sum_{k=1}^{m} 2^{k} d_{k} \leqq A \sum_{k=1}^{m}\left(n_{k}-n_{k-1}\right) \leqq A n_{m} \leqq A W_{D}\left(Q_{m}, Q_{0}\right) \leqq C \log \left(2+\frac{1}{l\left(Q_{n_{m}}\right)}\right) \leqq C m .
$$

Hence

$$
2^{N} \sum_{k=1}^{N} d_{k}=\sum_{k=1}^{N} 2^{k} d_{k}+\sum_{m=1}^{N-1}\left(2^{N-m-1} \sum_{k=1}^{m} 2^{k} d_{k}\right) \leqq C 2^{N} .
$$

Thus $d\left(Q, Q_{0}\right) \leqq A \sum_{k=1}^{N} d_{k} \leqq C$ which implies the assertion.

Q. E.D.

Applying Theorem 1 in case of $D=\boldsymbol{R}^{2}$ and applying Theorem 2 to the functions $F(Q)=\phi\left(Q, Q_{0}\right)$ or $F(Q)=\log \left(2+(1 / l(Q))\right.$ in case of $D \neq \boldsymbol{R}^{2}$, we have

COROLLARY 4. The following conditions are equivalent for a domain $D$

(1) $D$ is an inner NT A domain.

(2) Let $\phi$ be a $L^{\infty}(D)$ function on $D$ satisfying the condition

$$
\frac{1}{m(Q)} \int_{Q}\left|\phi-\phi_{Q}\right| d m \leqq \frac{M}{\phi\left(Q, Q_{0}\right)}, \quad Q \in \mathcal{A}(D)
$$

for some constant $M \geqq 0$, then $\varphi$ is a $B M O(D)$ multiplier.

(3) Let $\varphi$ be a $L^{\infty}(D)$ function on $D$ satisfying the same condition as (2) then $\varphi$ is a $\operatorname{VMO}(D)$ multiplier.

COROLLARY 5. The following conditions are equivalent for a domain $D$

(1) $D$ is a Holder domain.

(2) Let $\varphi$ be a $L^{\infty}(D)$ function on $D$ satisfying the condition

$$
\frac{1}{m(Q)} \int_{Q}\left|\phi-\phi_{Q}\right| d m \leqq M\left(\log \left(2+\frac{1}{l(Q)}\right)\right)^{-1}, \quad Q \in \mathcal{A}(D)
$$

for some constant $M \geqq 0$, then $\varphi$ is a $B M O(D)$ multiplier.

(3) Let $\varphi$ be a $L^{\infty}(D)$ function on $D$ satisfying the same condition as (2) then $\varphi$ is a $\operatorname{VMO}(D)$ multiplier.

\section{REFERFNCES}

[1] L. BROWN AND A. L. SHIELDS, Multipliers and cyclic vectors in the Bloch space, Michigan Math. J. 38 (1991), 141-146.

[2] F.W. GEHRING, Uniform domains and the Ubiquitous Quasidisk, Jahresber. Deutsch. Math.-Verein., 89 (1987), 88-103.

[3] Y. GOTOH, BMO extension theorem for relative uniform domains, J. Math. 
Kyoto Univ., to appear.

[4] S. Janson, On functions with conditions on the mean oscillation, Ark. Mat., 14 (1976), 189-196.

[5] P. JONES, Extension theorems for BMO, Indiana Univ. Math. J., 29 (1980), 41-66.

[6] E. NAKAI AND K. YABUTA, Pointwise multipliers for functions of bounded mean oscillation, J. Math. Soc. Japan, 37 (1985), 207-218.

[7] H. M. REIMANN AND T. RYCHENER, Funktionen beschränkter mittelerer Oszillation, Lecture Notes in Math. 489, Springer, 1975.

[ 8 ] K. Shimomura, A characterization of the inner NTA domain by the quasi-hyperbolic metric, to appear.

[9] D. A. STEGENGA, Bounded Toeplitz operators on $H^{1}$ and applications of duality between $H^{1}$ and the functions of bounded mean oscillation, Amer. J. Math., 98 (1976), 573-589.

DEPARTMENT OF MATHEMATICS,

KYOTO UNIVERSITY 